\section{pH Dependent Isotropic to Nematic Phase Transitions in Graphene Oxide Dispersions Reveal Droplet Liquid Crystalline Phases}

\author{
Rachel Tkacz ${ }^{\mathrm{a}}$, Rudolf Oldenbourg ${ }^{\mathrm{b}}$, Shalin B. Mehta ${ }^{\mathrm{b}}$, \\ Morteza Miansari ${ }^{\mathrm{a}}$, Amitabh Verma ${ }^{\mathrm{b}}$, Mainak \\ Majumder*a
}

Size fractionation, amplified by the surface charge density of graphene oxide (GO) sheets, broadens the $\mathrm{pH}$ dependent isotropic (I) to nematic $(\mathrm{N})$ phase transition in aqueous dispersions of graphene oxide (GO). In this biphasic region, a highly organized droplet nematic phase of uniform size $(20 \pm 2.8 \mu \mathrm{m}$ diameter) with an isotropic interior is observed.

Suspensions of 2D sheet-like materials exhibit transition from a disordered isotropic (I) phase to ordered nematic (N) phase. As noted by Onsager, ${ }^{1}$ despite their loss of orientational entropy, the $\mathrm{N}$ phase, is stabilized by a gain in excluded volume (or configurational) entropy. Graphene - a single atom thick layer of carbon atoms is receiving immense attention owing to the combination of properties such as large intrinsic mobility of electrons, massive surface area, immense mechanical strength and large thermal conductivity. Although significant progress has been made in the solid-state synthesis of graphene, liquid-phase graphene possess opportunities for large-scale synthesis and novel mesophases that may lead to ordered macroscopic structures such as thin films and fibers with enhanced electrical, mechanical and optical properties. ${ }^{2,3}$ Graphene solvated in chlorosulfonic acid ${ }^{2}$ and stabilized by surfactant ${ }^{4}$ exhibit liquid crystalline behavior, as well as their composites with discotic molecules. ${ }^{5}$ Chemically oxidized graphene sheets or graphene-oxide (GO) suspensions in water, the most accessible precursor to graphene-based materials, not surprisingly demonstrate I-N phase transitions, too. ${ }^{6-10}$ These studies have shown that the nematic phases of graphene exhibit 'brush-like' texture in massively organized
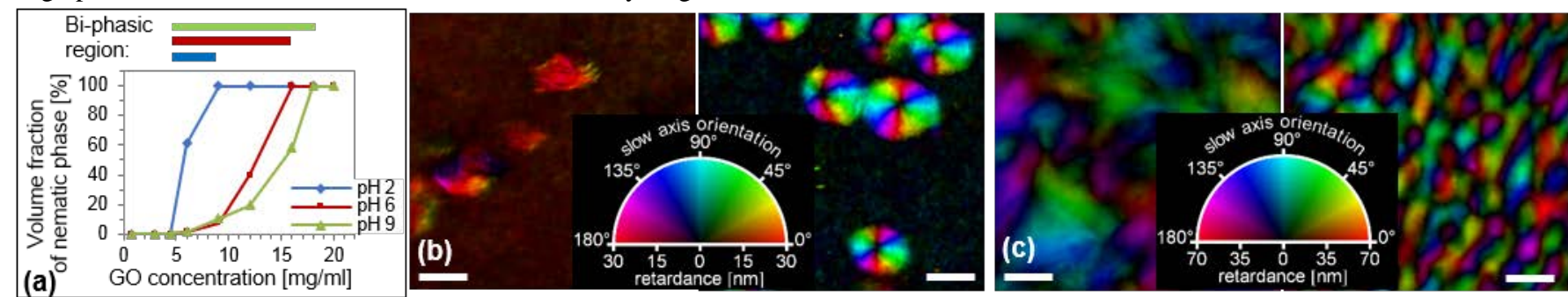

Figure 1. (a)Volume fraction of nematic phase versus concentration at different $\mathrm{pH}$ for GO dispersions in water. Pronounced broadening of the I-N biphasic region with increasing $\mathrm{pH}$ is noticeable. (b)-(c) (b)-(c) LC-PolScope processed images displaying the azimuth and retardance.. The color legend is a guide relating hue to azimuth (expressed around the circumference of the semi-circle) and brightness to retardance (expressed radially in nm). Typical morphology of: (b) metastable structures [6 mg/ml, $\mathrm{pH} 2$ (left) and $12 \mathrm{mg} / \mathrm{ml}$, pH 6 (right)], and (c) completely separated nematic phase [at pH 2 (left) and pH 6 (right)]. Scale bars are $20 \mu \mathrm{m}$. 
specimen. Note that the birefringence measurement is affected by the diattenuation, but the diattenuation is sensitive only to differential transmittance, as detailed in Mehta et $a .^{23}$ and demonstrated for reduced-GO in Tkacz et al. ${ }^{24}$ In the two extreme cases of $\mathrm{pH} 1$ and 14 , the suspensions did not form liquid crystals at any concentration due to aggregation of GO platelets (see S2 in ESI for further details). At pH 2, 6, and 9, a complete phase separation was observed after 46 days, with I phase at the top and $\mathrm{N}$ phase at the bottom of the capillary. As shown in Figure 1a, the ordered $\mathrm{N}$ phase starts to form at $\sim 5 \mathrm{mg} / \mathrm{ml}$ for each of these cases, the bi-phasic $(\mathrm{I}+\mathrm{N})$ region is present at intermediate concentrations and this region broadens with increasing $\mathrm{pH}$. Fully nematic ( $\mathrm{N}$ only) phases are obtained above 16 and $18 \mathrm{mg} / \mathrm{ml}$ for $\mathrm{pH} 6$ and 9 compared to $9 \mathrm{mg} / \mathrm{ml}$ at $\mathrm{pH} 2$. Figures 1b-c show the retardance and azimuth images of liquid crystalline domains in the bi-phasic region formed after 1 day and after complete separation of the I and $\mathrm{N}$ phase, at pHs 2 and 6, respectively (for images at $\mathrm{pH} 9$, please see S3 in ESI). Physically, the retardance images signify the distribution of birefringence while the azimuth images reflect the distribution of the slow axis of propagation. The 'brush-like' texture of the nematic phase at $\mathrm{pH} 2$, seen in figure 1 (c-left) is similar to previous reports. ${ }^{6,8,10} \mathrm{But}$ surprisingly, at $\mathrm{pH} 6$ and 9 of the bi-phasic region, the nematic domains with a droplet-like near perfect spherical shape is observed for the first time as shown in figure 1(b- right).

The I-N phase transition in ideal hard platelet system is governed by the ratio of thickness (t) to the diameter (D), i.e., $\frac{t}{\mathrm{D}}$. Thus, the concentration of onset of the I-N transition $\left(\Phi_{\mathrm{I}, \mathrm{V}}\right)$, as well as completion $\left(\Phi_{\mathrm{N}, \mathrm{v}}\right)$ scales with $\frac{t}{\mathrm{D}}$. Naturally, a polydispersity of the physical dimensions will lead to broadening of the bi-phasic region. ${ }^{25,26}$ Unlike monodisperse systems, polydispersity of dimensions also gives rise to osmotic forces from smaller particles that causes larger particles to come closer to each other driven by depletion interactions. ${ }^{27,28}$ Thus, polydispersity of dimensions asides from broadening the biphasic region can also fractionate the platelets. This fractionation mechanism has been experimentally observed by van der Kooij et al. ${ }^{25}$ and Wensink and Vroege ${ }^{29}$ for polydispersity in thickness where thinner particles separate to form a nematic phase, leaving the thicker (with large $\frac{t}{\mathrm{D}}$ ratio) ones to accumulate in the isotropic phase. A depletion mechanism was also observed by Zanchetta et al. ${ }^{30}$ during formation of liquid crystals in mixtures of single and double- strands DNA. In our case the experiments were performed with a large polydispersity in size of the GO sheets and we vary the $\mathrm{pH}$ which regulates the surface charge on the platelets. The osmotic pressure, $P$, between two planar surfaces depend upon the surface charge density, $\sigma$, according to $\approx \frac{2 \sigma^{2} e^{-\kappa d}}{\varepsilon \varepsilon_{0}}$, where $\kappa^{-1}$ is the Debye length, $d$ is the distance between the surfaces and $\varepsilon, \varepsilon_{0}$ are the permittivity constants. ${ }^{28} \mathrm{We}$ hypothesized that the broadening of the biphasic region is based on fractionation of the lateral width of the GO sheets, driven by surface charges since one can assume the GO sheets to have uni-layer thickness of $0.7-0.8 \mathrm{~nm} .{ }^{10,20}$ We argue that since the small GO sheets have higher surface charge density than larger sheets the osmotic pressure between them is increased and therefore the depletion, or the crowding of the large sheets, is enhanced. A qualitative illustration of the oxidative states of GO sheets at $\mathrm{pH}$ of 2, 6 and 9 and the charge density comparison between large and small sheets are shown in S4 in the ESI. As was suggested by Lerf and Klinowski ${ }^{12}$ the basal plane on GO contains aromatic domains along with oxidized domains rich in hydroxyls and epoxy groups, while the edges are decorated with carboxylic groups, which would provide GO sheets a negative charge. In a polydisperse GO suspension, the charged edges will also yield polydispersity in surface charge density because smaller sheets would have higher surface charge density than larger sheets and will increase with increasing $\mathrm{pH}$ in the range of 2-9. Without breaking this trend, we note that at $\mathrm{pH} 9$ chemical changes of GO may occur, as observed by darkening of the suspension and by UV-vis spectrometry (see S5 in ESI). To test the hypothesis of fractionation, the number-average diameter $\left(D_{n}\right)$ of the sheets in the isotropic and nematic phases at $\mathrm{pH}$ 2 and 6 was evaluated by quantitative analysis from scanning electron microscopy images (SEM, see S6 in ESI) of at least 500 sheets in each phase as shown in Figure 2 . We obtained $D_{n}$ of 390 $\mathrm{nm} \pm 59 \%$ and $430 \mathrm{~nm} \pm 71 \%$ for the I and $\mathrm{N}$ phases of $\mathrm{pH} 2$, and 330 $\mathrm{nm} \pm 51 \%$ and $515 \mathrm{~nm} \pm 76 \%$ for the $\mathrm{I}$ and $\mathrm{N}$ phases of $\mathrm{pH} 6$, respectively. It is clear from Figure 2 that the fractionation is enhanced at $\mathrm{pH} 6$ compared to $\mathrm{pH} 2$ and is consistent with our hypothesis for the broadening of the I-N phase transition.

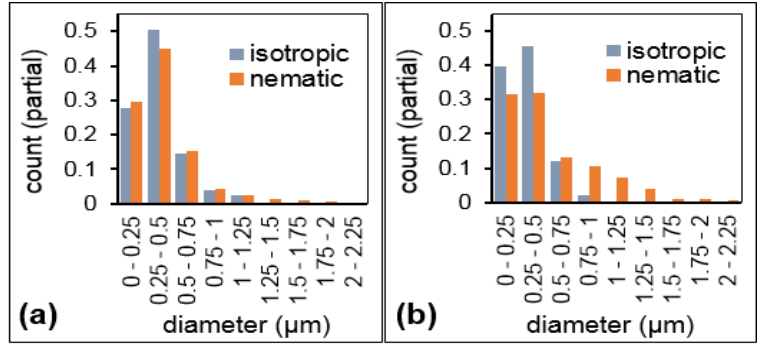

Figure 2. Size distribution of GO sheets in the isotropic and nematic phases at $\mathrm{pH} 2$ (a) and $\mathrm{pH} 6$ (b).

The droplets gradually merge to form a percolating 'worm-like' texture (images depicting the merging process are in S7 of ESI) and subsequently settles gravitationally to the bottom of the capillary as full $\mathrm{N}$ phase (Figure 1c-right). Liquid crystalline droplets of polymeric mesogens dispersed in continuous polymer phases have been investigated previously, but recently these droplets have demonstrated remarkable orientational sensitivity to interfacial interactions. ${ }^{31}$ Our spherical nematic droplets have a radial structure (Figure 3 ) where the director field $\hat{n}$ is orthogonal to the droplet surface, or in other words, the GO basal plane is tangential to the surface (Figure 3d top) - the easy direction of orientation imposed by surface tension forces. Figure $3 b$ shows the orientation of the slow axis measured with the birefringence mode and Figure $3 c$ the orientation of high transmittance direction measured using the diattenuation mode. These two orientations are perpendicular to each other as expected from the optical characteristics of GO, having higher refractive index and higher absorbance along the basal plane. The domain has polar symmetry with a void at the center of the droplet, remarkable uniformity in diameter $(20 \mu \mathrm{m} \pm 14 \%$ obtained from 130 measurements) and an average apparent retardance of $\sim 20$ $\mathrm{nm}$. The 2D order parameter, S, was calculated from azimuth distribution on the cross sections of the spherical domains. from $\sim 300$ pixels radially. Using $S=\left\langle 2 \cos ^{2} \theta-1\right\rangle$, where $\theta$ is the angle between the director and the normal of the GO sheets yields $S=0.99$ for both $\mathrm{pH} 6$ and 9. The content of the void in the center of the nematic droplet is rather intriguing. We know from birefringence (Figure 3a) and diattenuation (Figure 4a) measurements that the center of the droplet is less anisotropic than the periphery. However this could result from the presence of either isotropic GO, water or air. To resolve this issue, we looked at the average transmittance near the center, which is inversely proportional to the concentration of GO. As shown in Figure 4b, the average transmittance at the center of the droplet is similar to the transmittance at the periphery. Thus, we can conclude that the concentration of GO is similar to the isotropic or the liquid crystalline phase. 

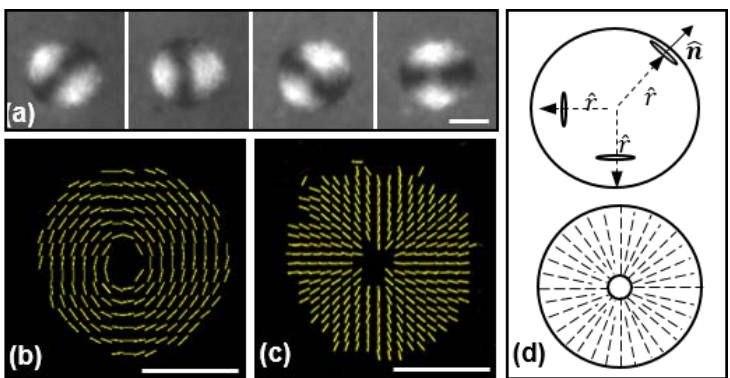

Figure 3. (a) Polarized light images of GO liquid crystalline droplet formed at $\mathrm{pH} 6$ using elliptically polarized light with $45^{\circ}$ difference in the polarization direction. (b) birefringence images displaying the orientation of the slow axis, (c) diattenuation images showing the orientation of high transmittance, (d) schematic illustration of the nematic director $\hat{n}$ in the droplet with regard to its radius $\hat{r}$ (top) and the radial structure of the director field (bottom). Scale bars are 10 $\mu \mathrm{m}$.

Furthermore, we examined the role of sonication in the formation of the droplet structure to verify that it is not arising from anchoring of GO sheets around a gas bubble (air or water vapors). We found out that the nematic droplets are formed even with no sonication at all (see S9 in ESI). Because the nematic droplets are metastable, we tracked their formation path (shown in S8 in ESI). An initially isotropic suspension starts to phase separate within the first two hours (top row). Later, the nematic domains are rearranged into spherical droplets with tangential alignment of the GO sheets, followed by a second phase separation, where an isotropic phase is formed at the center of each droplet.
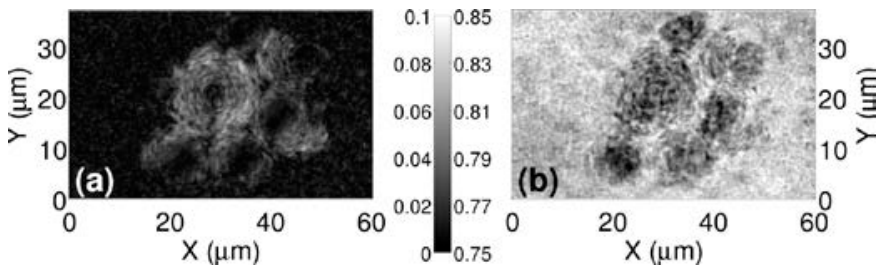

Figure 4. Diattenuation (a) and transmittance (b) of GO droplets showing that the isotropic center contains GO. The grayscale in the center shows that diattenuation varies from 0 to 0.1 , whereas the transmittance with respect to clear background varies from $75 \%$ to $85 \%$ indicating presence of GO. The diameter of the isotropic center of GO droplet is at least $4 \mu \mathrm{m}$, whereas depth of focus of the microscope is $~ 1.6 \mu \mathrm{m}$.

The average diameter of the isotropic phase inside each droplet is several micron and is therefore well above the resolution limit of the microscope optics $(\lambda /(2 \mathrm{NA})=0.37 \mu \mathrm{m})$. These phase separations are followed by annealing of the GO sheets inside the droplets (Figure S7, bottom row), which we presume occurs due to anchoring at the droplet interface and a directionality imposed by the surface tension. ${ }^{33}$ However further study is required for thoroughly resolving the formation mechanism of these droplet nematic phases.

\section{Conclusions}

In this communication we report the $\mathrm{pH}$ dependent phase behavior of aqueous dispersions of GO for the first time. The studies reveal two major findings that are of interest. Firstly, isotropic to nematic phase transitions in these suspensions are governed by not only polydispersity of dimensions, but also polydispersity of surface charges. These two coupled phenomena enhances, particularly at $\mathrm{pH}>\mathrm{pKa}$ of the carboxylic acid groups on GO, the depletion interaction to segregate large graphene (515 $\mathrm{nm} \pm 76 \%)$ sheets into the nematic phase and smaller (330 $\mathrm{nm} \pm 51 \%$ ) into the isotropic. This observation may provide a solution to size-separation of graphene sheets that has proven to be quite difficult. Secondly, we report hitherto unobserved spherical droplet nematic phases of GO suspended in isotropic GO and because larger GO sheets are more hydrophobic than smaller GO sheets ${ }^{16}$, this droplet structure bears close structural analogy to oil in water emulsions.

\section{Notes and references}

${ }^{a}$ Nanoscale Science and Engineering Laboratory (NSEL), Mechanical and Aerospace Engineering Department, Monash University, Clayton, VIC 3800, Australia

${ }^{b}$ Marine Biological Laboratory, Woods Hole MA 02543, and Physics Department, Brown University, Providence RI 02912, USA

*Email: mainak.majumder@monash.edu; Tel: +61399056255

†Electronic Supplementary Information (ESI) available: Experimental procedures, UV-vis spectra and polarized light characterization see DOI: $10.1039 / \mathrm{c} 000000 \mathrm{x} /$

Acknowledgements: Supports from the Australian Research Council (LP110100612 to MM), National Institute of Biomedical Imaging and Bioengineering (R01EB002045 to RO) and HFSP fellowship (to SM) are acknowledged.

1. L. Onsager, Ann. N. Y. Acad. Sci., 1949, 51, 627-659.

2. N. Behabtu, J. R. Lomeda, M. J. Green, A. L. Higginbotham, A. Sinitskii, D. V. Kosynkin, D. Tsentalovich, A. N. G. Parra-Vasquez, J. Schmidt, E. Kesselman, Y. Cohen, Y. Talmon, J. M. Tour and M. Pasquali, Nat Nano, 2010, 5, 406-411.

3. Z. Xu and C. Gao, Nat. Commun., 2011, 2, 571.

4. C. Zamora-Ledezma, N. Puech, C. Zakri, E. Grelet, S. E. Moulton, G. G. Wallace, S. Gambhir, C. Blanc, E. Anglaret and P. Poulin, J.Phys.Chem.Lett., 2012, 3, 2425-2430.

5. A. B. Shivanandareddy, S. Krishnamurthy, V. Lakshminarayanan and S. Kumar, Chem. Commun., 2014, 50, 710-712.

6. J. E. Kim, T. H. Han, S. H. Lee, J. Y. Kim, C. W. Ahn, J. M. Yun and S. O. Kim, Angew. Chem. Inter Ed., 2011, 50, 3043-3047.

7. S. H. Aboutalebi, M. M. Gudarzi, Q. B. Zheng and J.-K. Kim, Adv. Funct. Mater., 2011, 21, 2978-2988.

8. B. Dan, N. Behabtu, A. Martinez, J. S. Evans, D. V. Kosynkin, J. M. Tour, M. Pasquali and I. I. Smalyukh, Soft Matter, 2011, 7, 11154-11159. 9. F. Guo, F. Kim, T. H. Han, V. B. Shenoy, J. Huang and R. H. Hurt, ACS Nano, 2011, 5, 8019-8025.

10. Z. Xu and C. Gao, ACS Nano, 2011, 5, 2908-2915.

11. D. Li, M. B. Muller, S. Gilje, R. B. Kaner and G. G. Wallace, Nat Nano, 2008, 3, 101-105.

12. A. Lerf, H. He, M. Forster and J. Klinowski, J.Phys.Chem.B 1998, 102, 4477-4482.

13. J. Kim, L. J. Cote, F. Kim, W. Yuan, K. R. Shull and J. Huang, J. Am. Chem. Soc., 2010, 132, 8180-8186.

14. B. Konkena and S. Vasudevan, J.Phys.Chem.Lett., 2012, 3, 867-872.

15. C.-J. Shih, S. Lin, R. Sharma, M. S. Strano and D. Blankschtein, Langmuir, 2011, 28, 235-241.

16. X. Hu, Y. Yu, W. Hou, J. Zhou and L. Song, Appl. Surf. Sci., 2013, 273, 118-121.

17. L. J. Cote, F. Kim and J. Huang, J. Am. Chem. Soc., 2008, 131, 10431049. 
18. M. Sametband, U. Shimanovich and A. Gedanken, New J. Chem., 2012, 36, 36-39.

19. W. S. Hummers and R. E. Offeman, J. Am. Chem. Soc., 1958, 80, 1339-1339.

20. N. I. Kovtyukhova, P. J. Ollivier, B. R. Martin, T. E. Mallouk, S. A. Chizhik, E. V. Buzaneva and A. D. Gorchinskiy, Chem. Mater., 1999, 11, 771-778.

21. R. Oldenbourg and G. Mei, J.Microsc. 1995, 180, 140-147.

22. R. Oldenbourg, G. Harris, A. Verma and S. B. Mehta, http://openpolscope.org/pages/Birefringence.htm, 2013.

23. S. B. Mehta, M. Shribak and R. Oldenbourg, J. Opt., 2013, 15, 094007.

24. R. Tkacz, R. Oldenbourg, A. Fulcher, M. Miansari and M. Majumder, J.Phys.Chem.C, 2013, 118, 259-267.

25. F. M. van der Kooij, D. van der Beek and H. N. W. Lekkerkerker, J.Phys.Chem. B, 2001, 105, 1696-1700.

26. M. A. Bates and D. Frenkel, J.Chem.Phys, 1999, 110, 6553-6559.

27. P. Nelson, Biological Physics, Energy, Information, Life, 1st ed., Clancy Marshall, New York, 2008.

28. J. N. Israelachvili, Intermolecular and Surface Forces 3edn., Elsevier Inc, 2011.

29. H. H. Wensink and G. J. Vroege, Phys.Rev.E., 2002, 65, 031716031711.

30. G. Zanchetta, M. Nakata, M. Buscaglia, T. Bellini and N. A. Clark, Proc. Natl. Acad. Sci. U.S.A.,2008, 105, 1111-1117.

31. D. S. Miller, X. Wang and N. L. Abbott, Chem. Mater., 2013, 26, 496-506.

32. P. G. de Gennes and J. Prost, The physics of liquid crystals, 2nd ed. Oxford University Press 1993. 